\title{
CINÉTICA DE SECAGEM DE TOMATES DO TIPO CEREJA (Lycopersicon esculentum var. cerasiforme) E UVA (Lycopersicon esculentum Mill.) UTILIZANDO SECADOR DE BANDEJAS
}

\author{
H. O. N. ALTINO ${ }^{1}$ e R. N. CUNHA ${ }^{1}$ \\ ${ }^{1}$ Centro Universitário de Patos de Minas, Departamento de Engenharia Química \\ E-mail para contato: heitor.engquimica@gmail.com
}

\begin{abstract}
RESUMO - O Brasil atua como um dos maiores produtores mundiais de tomates. Todavia, apesar da elevada produção, as perdas durante a cadeia produtiva variam entre $20 \%$ e $50 \%$. Assim, novas tecnologia buscam reduzir tais perdas, destacandose a secagem para produção de tomates secos. Os tomates tipo cereja e uva apresentam potencial para a produção de tomate seco, devido à abundância, qualidades sensoriais e facilidades operacionais. Neste contexto, o objetivo principal desse trabalho foi estudar a cinética de secagem dos tomates tipo cereja e uva. Utilizou-se um secador de bandejas sob as temperaturas de $60^{\circ} \mathrm{C}, 70^{\circ} \mathrm{C}$ e $80^{\circ} \mathrm{C}$. Além disso, aplicou-se diversos modelos matemáticos para a descrição das curvas de secagem. Constatou-se que os modelos avaliados são aplicáveis aos dados cinéticos, sendo que o modelo de Midilli et al. mostrou-se o mais indicado para descrição do processo. Observou-se ainda pequenos intervalos de taxa de secagem constante, indicando que a cinética do processo é controlada pela difusão da água pela estrutura do material. Os resultados obtidos demonstram a viabilidade operacional da secagem de tomates do tipo cereja e uva em secador de bandejas.
\end{abstract}

\section{INTRODUÇÃO}

Conforme o Instituto Brasileiro de Geografia e Estatística (2015), o Brasil possui uma área plantada de 55.016 ha e uma produção de 3.467.990 toneladas de tomate, resultando em um rendimento de $63.036 \mathrm{~kg} / \mathrm{ha}$. Apesar de elevada produção, as perdas variam entre $20 \%$ e $50 \%$ devido à safra, doenças, sistema de colheita, manuseio e comercialização (Pacco, 2008). Assim, novos processos de industrialização vêm sendo pesquisados, visando a redução dessas perdas, destacando-se a secagem para produção de tomates secos (Mata; Branga; Kross, 2003).

Os tomates tipo cereja e uva apresentam grande potencial para a produção de tomate seco, devido à abundância, coloração vermelho brilhante, sabor intenso e facilidades operacionais (May, 2004; Azoubel; Murr, 2000). No âmbito acadêmico, são poucas as pesquisas envolvendo a secagem de tomates do tipo cereja e uva. Todavia, Orlandin, Fontana e Sandri (2010) já comprovaram a aceitação desse primeiro tipo por análise sensorial, após a desidratação osmótica, e May (2004) registrou uma patente para a secagem ao sol desse segundo tipo.

Com base nessa problemática, o objetivo desse trabalho foi estudar a cinética de secagem dos tomates tipo cereja e uva. De forma que, os dados obtidos possam auxiliar na projeção de 
equipamentos, do processo de secagem, contribuindo, no futuro, para o desenvolvimento de produtos, nutritivos e econômicos, que possam ser inseridos no mercado brasileiro.

\section{MATERIAS E MÉTODOS}

\subsection{Matéria-Prima}

Os materiais adotados como objeto de estudo do presente trabalho (tomates do tipo cereja e uva) foram obtidos no comércio local da cidade de Patos de Minas, Minas Gerias, Brasil. A seleção foi realizada atentando-se para frutos sadios, coloração avermelhada, uniformidade, ausência de pedúnculo, desenvolvimento fisiológico, limpeza, textura de polpa firme, ausência de danos mecânicos, fisiológicos, pragas e doenças. Em seguida, $2000 \mathrm{~g}$ de tomates foram limpos com água deionizada (CS1800, Permution ${ }^{\circledR}$, Brasil) e sanitizados com uma solução de cloro ativo a $0,5 \%$ durante $30 \mathrm{~min}$. Após esse período de tempo, os tomates foram cortado perpendicularmente ao eixo principal e gravitacionalmente escorridos durante $15 \mathrm{~min}$.

\subsection{Caracterização Físico-Química}

A umidade inicial foi estimada por um Analisador de Umidade por Infravermelho (IV 2500, Gehaka ${ }^{\circledR}$, Brasil) com taxa de Auto Dry de $1 \% \cdot \mathrm{min}^{-1}$ e temperatura de $105^{\circ} \mathrm{C}$. A análise granulométrica foi realizada por medição dos três eixos principais e pesagem das amostras. As medições foram realizadas por meio de um paquímetro digital $\left(316119\right.$, MTX $^{\circledR}$, Brasil) e as pesagens por meio de uma balança digital (BG-440, Gehaka ${ }^{\circledR}$, Brasil).

\subsection{Sistema Experimental}

Os experimentos forma conduzidos em um secador de bandejas (PE 60, Pardal ${ }^{\circledR}$, Brasil), como esquematicamente mostrado na Figura 1. O equipamento contava com bandejas de secagem $(0,5 \mathrm{~m} \times 0,82 \mathrm{~m})$ de plástico para alimentos com bordas de aço para o suporte das amostras, área de secagem de $0,36 \mathrm{~m}^{2}$, volume de $0,30 \mathrm{~m}^{3}$, velocidade do ar de secagem de 0,11 $\pm 0.02 \mathrm{~m} \cdot \mathrm{s}^{-1}$ e potência de $1,3 \mathrm{~kW} \cdot \mathrm{h}^{-1}$. Os experimentos forma conduzidos nas seguintes condições ambientais: umidade do ar 44,88 $\pm 1,92 \%$, temperatura do ar $26,69 \pm 0,41^{\circ} \mathrm{C}$ e pressão atmosférica de 91.580,47 $\pm 137,32 \mathrm{~Pa}$.

Figura 1 - Sistema experimental.

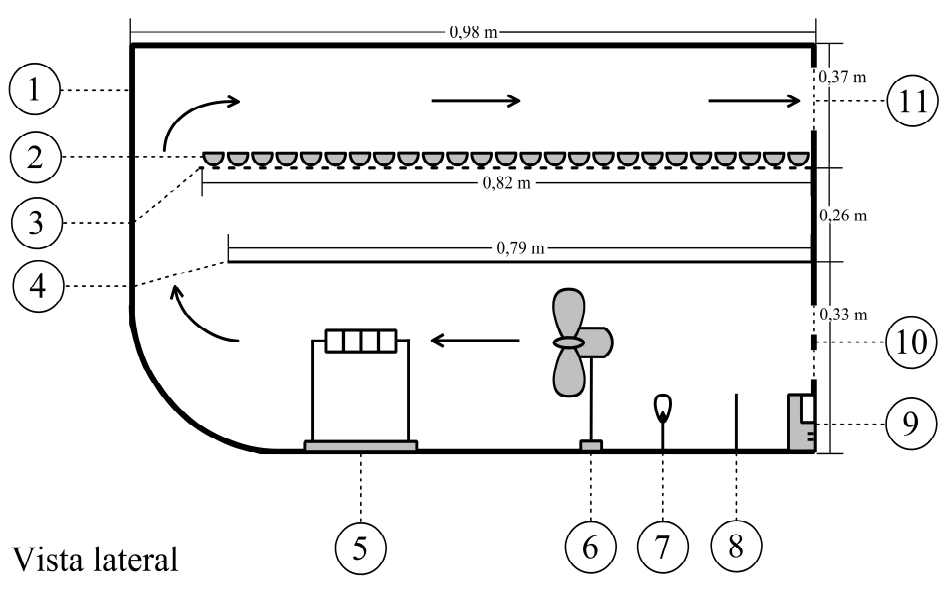

Legenda

1 - Secador de bandejas

2 - Camada de tomates

3 - Bandeja de secagem

4 - Bandeja de proteção

5 - Resistência termica

6 - Soprador

7 - Lâmpada

8 - Termopar

9 - Controlador indicador de temperatura

10 - Entrada de fluxo de ar

11 - Saída de fluxo de ar 


\subsection{Estudos Cinéticos}

Os estudos cinéticos foram realizados em um secador de bandejas, conforme descrito na seção 2.3. As temperaturas de secagem foram $60^{\circ} \mathrm{C}, 70^{\circ} \mathrm{C}$ e $80^{\circ} \mathrm{C}$ para os tomates tipo cereja e uva. Os tomates foram distribuídos uniformemente na bandeja de secagem com a face de corte para cima, formando uma camada. Durante o processo de secagem os tomates foram pesados

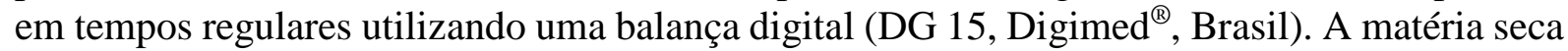
foi mensurada, após a umidade de equilíbrio ser atingida, aumentando-se a temperatura de secagem para $105^{\circ} \mathrm{C}$ até se alcançar peso constante.

A umidade total $\left(X_{t}\right)\left(\mathrm{g} \mathrm{H}_{2} \mathrm{O} \cdot \mathrm{g}^{-1}\right.$ matéria seca) foi calculada por meio da Equação 1, a umidade livre $(X)\left(\mathrm{g} \mathrm{H}_{2} \mathrm{O} \cdot \mathrm{g}^{-1}\right.$ matéria seca) pela Equação 2 e a taxa de secagem $(R)\left(\mathrm{g} \cdot \mathrm{min}^{-1} \mathrm{~m}^{-}\right.$ ${ }^{2}$ ) empregando-se a Equação 3 (Geankoplis, 1993).

$$
\begin{aligned}
& X_{t}=\frac{W-W_{s}}{W_{s}} \\
& X=X_{t}-X^{*} \\
& R=-\frac{L_{s}}{A} \frac{d X}{d t}
\end{aligned}
$$

Em que $W$ é a massa total do sólido $(\mathrm{g}), W_{s}$ a massa de sólido seco a $105^{\circ} \mathrm{C}(\mathrm{g}), X^{*}$ a umidade de equilíbrio ( $\mathrm{g} \mathrm{H}_{2} \mathrm{O}_{\mathrm{g}} \mathrm{g}^{-1}$ matéria seca), $t$ o tempo (min), $L_{s}$ a massa de sólido seco utilizada $(\mathrm{g})$ e $A$ a área superficial do material exposta a secagem $\left(\mathrm{m}^{2}\right)$.

\subsection{Modelagem Matemática da Cinética de Secagem}

Com o objetivo de descrever os fenômenos envolvidos no processo de secagem dos tomates tipo cereja e uva, buscou-se aplicar modelos matemáticos para descrição das curvas de secagem, os quais podem ser visualizados na Tabela 1.

Tabela 1 - Modelos matemáticos para descrição das curvas de secagem.

\begin{tabular}{llll}
\hline \multicolumn{1}{c}{ Designação } & \multicolumn{1}{c}{ Modelo } & \multicolumn{1}{c}{ Referência } & Eq. \\
\hline Page & $M R=e^{-k t^{n}}$ & Karathanos e Belessiotis (1999) & $(4)$ \\
Midilli et al. & $M R=a e^{-k t^{n}}+b t$ & Midilli, Kucuk e Yapar (2002) & (5) \\
Henderson e Pabis & $M R=a e^{-k t}$ & Akpinar, Bicer e Yildiz (2003) & $(6)$ \\
Wang e Singh & $M R=1+a t+b t^{2}$ & Wang et al. (2007) & $(7)$ \\
Newton & $M R=e^{-k t}$ & O'Callaghan et al. (1971) & $(8)$ \\
\hline
\end{tabular}

$a$ e $b$ : constantes de secagem adimensional, $k, k_{0}$ e $k_{1}$ : constantes da cinética de secagem $\left(\mathrm{min}^{-1}\right), R U$ : razão de umidade, $n$ : constante de cinética de secagem adimensional e $t$ : tempo (min).

Tais modelos são expressos na forma de razão de umidade $(R U)$, como matematicamente mostrado pela Equação 9.

$$
R U=\frac{X_{t}-X^{*}}{X_{i}-X^{*}}
$$


Em que $X_{t}$ é a umidade total $\left(\mathrm{g} \mathrm{H}_{2} \mathrm{O} . \mathrm{g}^{-1}\right.$ matéria seca), $X_{i}$ a umidade total inicial $\left(\mathrm{g} \mathrm{H}_{2} \mathrm{O} . \mathrm{g}^{-}\right.$ ${ }^{1}$ matéria seca) e $X^{*}$ a umidade de equilíbrio $\left(\mathrm{g} \mathrm{H}_{2} \mathrm{O} \cdot \mathrm{g}^{-1}\right.$ matéria seca).

\section{RESULTADOS E DISCUSSÃO}

\subsection{Características Físico-Química}

A Tabela 2 apresenta as características físico-químicas dos tomates tipo cereja e uva. Para todos os parâmetros estudados é possível observar pequenos intervalos de confiança, indicando a semelhança entre as amostras e permitindo inferir que os efeitos observados nos estudos cinéticos não foram influenciados pelas características físico-químicas das amostras.

Tabela 2 - Características físico-químicas dos tomates tipo cereja e uva.

\begin{tabular}{lrr}
\hline \multicolumn{1}{c}{ Parâmetros } & Tomate cereja & \multicolumn{1}{c}{ Tomate uva } \\
\hline$X_{0}(\%)$ & $86,269 \pm 1,003$ & $84,797 \pm 0,909$ \\
$\rho\left({\left.\mathrm{g} \cdot \mathrm{cm}^{-3}\right)}_{V\left(\mathrm{~cm}^{3}\right)}\right.$ & $1,217 \pm 0,004$ & $1,219 \pm 0,004$ \\
$A E S\left(\mathrm{~cm}^{2} \cdot \mathrm{g}^{-1}\right)$ & $9,664 \pm 0,286$ & $6,263 \pm 0,110$ \\
$D P S(\mathrm{~cm})$ & $1,847 \pm 0,157$ & $2,465 \pm 0,122$ \\
$A S C\left(\mathrm{~cm}^{2}\right)$ & $2,687 \pm 0,286$ & $1,988 \pm 0,064$ \\
\hline$X_{i}: \mathrm{m}^{2} \mathrm{~d}$ & $1.893,693 \pm 379,114$ & $1.892,664 \pm 263,123$ \\
\hline
\end{tabular}

$X_{i}$ : umidade inicial (\%), $\rho$ : massa específica $\left(\mathrm{g} . \mathrm{cm}^{-3}\right), V:$ volume $\left(\mathrm{cm}^{3}\right), A E S$ : área específica superficial $\left(\mathrm{cm}^{2} \cdot \mathrm{g}^{-1}\right), D P S$ : diâmetro principal de Sauter $(\mathrm{cm})$ e $A S C$ : área superficial de corte $\left(\mathrm{cm}^{2}\right)$.

\subsection{Cinética de Secagem}

A Tabela 3 apresenta os resultados da aplicação dos modelos matemáticos nas curvas de secagem dos tomates tipo cereja e uva. Os valores dos coeficiente de determinação $\left(R^{2}\right)$ variaram de 0,9133 até 0,9997 para o tomate cereja e 0,9435 até 0,9992 para o tomate uva. Em todas as temperaturas, o modelo de Midilli et al. apresentou os maiores valores de $R^{2}$ para os tomates tipo cereja e uva. Indicando a capacidade do modelo para descrição dos fenômenos envolvidos na cinética de secagem desses materiais.

As curvas de secagem com o modelo de Midilli et al. aplicado podem ser vistas na Figura 2 (a) para o tomate cereja e na Figura 2 (c) para o tomate uva. Observa-se uma a rápida redução inicial do conteúdo de água, provavelmente devido a remoção da água livre. Após esse período, não há água livre suficiente e as interações entre a água e a estrutura do sólido podem começar a se intensificar, diminuindo o conteúdo de água. Tal redução cessa quando a umidade de equilíbrio é alcançada, resultando em um período constante de baixa razão de umidade.

O modelo de Midilli et al. foi proposto para descrever os fenômenos envolvidos na secagem de matérias biológicos, o que está de acordo com as curvas de taxa de secagem para o tomate tipo cereja (Figura 2 (b)) e uva (Figura 2 (d)) (Midilli et al., 2002). Nota-se curvas características de materiais biológicos, com pequenos intervalos de taxa constante. Indicando que a cinética do processo é controlada pela lenta difusão da água por entre a estrutura do material (Geankoplis, 1993). Outro efeito é o aumento das taxas de secagem com o aumento da temperatura. $\mathrm{O}$ que pode ser atribuído ao aumento do potencial de transferência de calor entre o ar e a estrutura dos tomates em altas temperaturas, facilitando a saída de água. 
Tabela 3 - Parâmetros dos modelos para descrição das curvas de secagem.

\begin{tabular}{|c|c|c|c|c|c|c|c|}
\hline \multirow{2}{*}{ Modelo } & \multirow{2}{*}{ Parâmetro } & \multicolumn{3}{|c|}{ Tomate cereja } & \multicolumn{3}{|c|}{ Tomate uva } \\
\hline & & $60^{\circ} \mathrm{C}$ & $70^{\circ} \mathrm{C}$ & $80^{\circ} \mathrm{C}$ & $60^{\circ} \mathrm{C}$ & $70^{\circ} \mathrm{C}$ & $80^{\circ} \mathrm{C}$ \\
\hline \multirow{3}{*}{ Page } & $k\left(\min ^{-1}\right)$ & 0,0006 & 0,0008 & 0,0009 & 0,0007 & 0,0008 & 0,0008 \\
\hline & $n$ & 1,2092 & 1,2104 & 1,2150 & 1,2087 & 1,2113 & 1,2191 \\
\hline & $R^{2}$ & 0,9984 & 0,9989 & 0,9971 & 0,9980 & 0,9978 & 0,9964 \\
\hline \multirow{5}{*}{ Midilli et al. } & $a$ & 0,9876 & 0,9844 & 0,9813 & 0,9819 & 0,9829 & 0,9839 \\
\hline & $k(\mathrm{mi}$ & 0,0006 & 0,0007 & 0,0008 & 0,0006 & 0,0007 & 0,0008 \\
\hline & $n$ & 1,2090 & 1,2149 & 1,2210 & 1,2141 & 1,2168 & 1,2186 \\
\hline & $b$ & $-1,60 \mathrm{E}-05$ & $-1,20 \mathrm{E}-05$ & $-1,30 \mathrm{E}-05$ & $-1,50 \mathrm{E}-05$ & $-1,64 \mathrm{E}-05$ & $-1,10 \mathrm{E}-05$ \\
\hline & $R^{2}$ & 0,9994 & 0,9997 & 0,9975 & 0,9992 & 0,9987 & 0,9965 \\
\hline \multirow{3}{*}{$\begin{array}{l}\text { Henderson e } \\
\text { Pabis }\end{array}$} & $a$ & 1,0522 & 1,0527 & 1,0695 & 1,0376 & 1,0630 & 1,0767 \\
\hline & $k\left(\min ^{-1}\right)$ & 0,0024 & 0,0028 & 0,0035 & 0,0025 & 0,0030 & 0,0033 \\
\hline & $R^{2}$ & 0,9912 & 0,9934 & 0,9885 & 0,9925 & 0,9905 & 0,9880 \\
\hline \multirow{3}{*}{ Wang e Singh } & $a$ & $-0,0016$ & $-0,0021$ & $-0,0017$ & $-0,0016$ & $-0,0017$ & $-0,0017$ \\
\hline & $b$ & 1,00E-06 & $1,00 \mathrm{E}-06$ & $1,00 \mathrm{E}-06$ & $1,00 \mathrm{E}-06$ & $1,00 \mathrm{E}-06$ & $1,00 \mathrm{E}-06$ \\
\hline & $R^{2}$ & 0,9963 & 0,9768 & 0,9133 & 0,9922 & 0,9619 & 0,9435 \\
\hline \multirow{2}{*}{ Newton } & $k\left(\min ^{-1}\right)$ & 0,0023 & 0,0027 & 0,0033 & 0,0024 & 0,0029 & 0,0030 \\
\hline & & 0,9881 & 0,9904 & 0,9841 & 0,9899 & 0,9864 & 0,9826 \\
\hline
\end{tabular}

Figura 2 - Dados experimentais para o tomate cereja a $60^{\circ} \mathrm{C}(\boldsymbol{\square}), 70^{\circ} \mathrm{C}(\boldsymbol{O})$ e $80^{\circ} \mathrm{C}(\boldsymbol{\Delta})$ : curva de secagem (a) e curvas de taxa de secagem (b). Dados experimentais para o tomate uva

$60^{\circ} \mathrm{C}(\mathbf{D}), 70^{\circ} \mathrm{C}(\boldsymbol{D})$ e $80^{\circ} \mathrm{C}(\Delta)$ : curva de secagem (c) e curvas de taxa de secagem $(\mathrm{d})$.
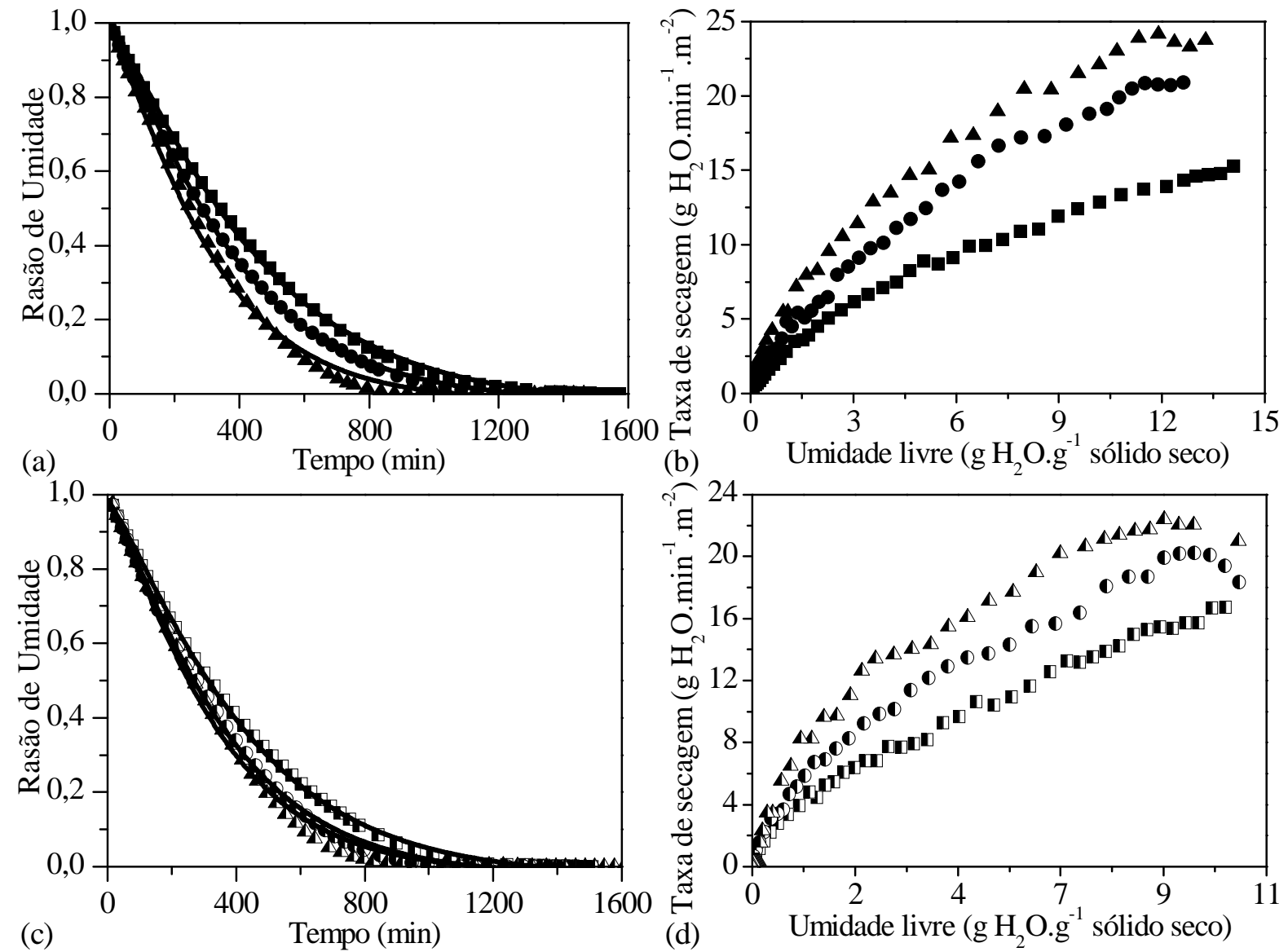


\section{CONCLUSÃO}

No presente trabalho foi realizado o estudo da cinética de secagem de tomates do tipo cereja e uva. Para tal propósito utilizou-se um secador de bandejas sob as temperaturas de ar de secagem de $60^{\circ} \mathrm{C}, 70^{\circ} \mathrm{C}$ e $80^{\circ} \mathrm{C}$. Além disso, aplicou-se diversos modelos matemáticos para a descrição das curvas de secagem. Constatou-se que os modelos avaliados são aplicáveis aos dados cinéticos, contudo o modelo de Midilli et al. se mostrou o mais recomendado para descrição do processo. Observou-se ainda pequenos intervalos de taxa de secagem constante, indicando que a cinética do processo é controlada pela lenta difusão da água por entre a estrutura do material. Os resultados obtidos demonstram a viabilidade operacional da secagem de tomates do tipo cereja e uva utilizando secador de bandejas.

\section{REFERÊNCIAS}

AKPINAR, E. K.; BICER, Y.; YILDIZ, C. Thin layer drying of red pepper. J. Food Eng., v. 59, p. 99-104, 2003.

AZOUBEL, P. M.; MURR, F. E. X. Mathematical modelling of osmotic dehydration of cherry tomato (Lycopersicon esculentum var. cerasiforme). Ciênc. Tecnol. Aliment., v. 20, p. 228-232, 2000.

GEANKOPLIS, C. J. Transport Processes and Unit Operations. New Jersey: Prentice-Hall, 1993.

INSTITUTO BRASILEIRO DE GEOGRAFIA E ESTATÍSTICA. Levantamento sistemático da produção agrícola. Rio de Janeiro: IBGE, 2015.

KARATHANOS, V. T.; BELESSIOTIS, V. G. Application of a Thin-Layer Equation to Drying Data of Fresh and Semi-dried Fruits. J. Agric. Eng. Res., v. 74, p. 355-361, 1999.

MATA, M. E. R. M. C.; BRAGA, M. E. D.; KROSS, R. K. Secagem osmótica de tomate: efeito da epiderme. Rev. Bras. de Prod. Agroindustriais, n. 1, p.77-84, 2003.

MAY, Edward. Tomato Raisin. US nº 6743560B2, 1 jun. 2004.

MIDILLI, A.; KUCUK, H.; YAPAR, Z. A New Model for Single-Layer Drying. Drying Technol., v. 20, p. 1503-1513, 2002.

O'CALLAGHAN, J. R., MENZIES, D. J., BAILEY, P. H. Digital simulation of agricultural drier performance. J. Agric. Eng. Res., v. 16, p. 223-244, 1971.

ORLANDIN, A.; FONTANA, R. C.; SANDRI, I. G. Estudo de pré-tratamentos na desidratação de tomate-cereja (Lycopersicon esculentum var. cerasiforme). Braz. J. Food Technol., v. 13, p. 226-231, 2010.

PACCO, H. C. Desenvolvimento de um sistema de bomba de calor águalágua para resfriamento e secagem de tomates. 2008. $204 \mathrm{f}$. Tese (Doutorado) - Curso de Engenharia Agrícola, Faculdade de Engenharia Agrícola, UNICAMP, Campinas, 2008.

WANG, Z.; SUN, J.; CHEN, F.; LIAO, X.; HU, X. Mathematical modelling on thin layer microwave drying of apple pomace with and without hot air pre-drying. J. Food Eng., v. 80, p. 536-544, 2007. 